\title{
The prognostic roles of platelet-to-lymphocyte ratio and neutrophil-to-lymphocyte ratio in gastrointestinal stromal tumours: a meta-analysis
}

\author{
Zhe-Wei Wei $^{1,2 \#}$, Wei-Bin Huang ${ }^{1,2 \#}$, Dong-Jie Yang ${ }^{1,2}$, Yu-Jie Yuan ${ }^{1,2}$, Yu-Long He ${ }^{1,2,3}$, Chang-Hua Zhang ${ }^{3}$ \\ ${ }^{1}$ Department of Gastrointestinal Surgery, the First Affiliated Hospital of Sun Yat-sen University, Guangzhou, China; ${ }^{2}$ Gastric Cancer Center, Sun \\ Yat-sen University, Guangzhou, China; ${ }^{3}$ Department of Digestive Diseases, the Seventh Affiliated Hospital of Sun Yat-sen University, Shenzhen, \\ China \\ Contributions: (I) Conception and design: YL He, CH Zhang; (II) Administrative support: ZW Wei, WB Huang; (III) Provision of study materials or \\ patients: WB Huang, DJ Yang; (IV) Collection and assembly of data: DJ Yang, WB Huang; (V) Data analysis and interpretation: YJ Yuan, ZW Wei; (VI) \\ Manuscript writing: All authors; (VII) Final approval of manuscript: All authors. \\ \#These authors contributed equally to this work. \\ Correspondence to: Chang-Hua Zhang, MD, PhD. Department of Digestive Diseases, the Seventh Affiliated Hospital of Sun Yat-sen University, 628 \\ Zhenyuan Road, Shenzhen, Guangdong 518000, China. Email: zhchangh@mail.sysu.edu.cn; Yu-Long He, MD, PhD. Gastric Cancer Center, Sun \\ Yat-sen University, 58 Zhongshan 2nd Road, Guangzhou, Guangdong 510080, China. Email: heyulong@mail.sysu.edu.cn.
}

\begin{abstract}
Background: The platelet-to-lymphocyte ratio (PLR) and neutrophil-to-lymphocyte ratio (NLR) have been found to be associated with prognosis in several solid tumours. However, the prognostic roles of PLR and NLR in gastrointestinal stromal tumours (GISTs) remain controversial. The aim of this meta-analysis was to assess the prognostic roles of PLR and NLR in GISTs.

Methods: We searched MEDLINE, EMBASE and the Cochrane Library for relevant articles. A systematic review was performed to calculate pooled hazard ratios (HRs) for disease-free survival (DFS) and overall survival (OS) by fixed-effects/random-effects models.

Results: Fourteen studies containing 3,151 subjects were finally enrolled in this meta-analysis. Eight studies including 2,560 patients investigated the prognostic effect of PLR, and thirteen studies with 2,751 subjects explored the prognostic effect of NLR. Both elevated PLR (HR: 1.29, 95\% CI: 1.10-1.52, P=0.002) and NLR (HR: 1.37, 95\% CI: 1.15-1.63, P=0.0005) were significantly associated with decreased DFS. The pooled HR for PLR was not significantly different from that for NLR. High PLR and NLR correlated with increased tumour sizes, more advanced tumour stages and mitotic index (>5/50 HPF). In addition, elevated PLR was related to adjuvant tyrosine kinase inhibitor (TKI) therapy.
\end{abstract}

Conclusions: Elevated preoperative PLR and NLR are associated with poor outcomes in patients with GISTs.

Keywords: Gastrointestinal stromal tumours (GISTs); platelet-to-lymphocyte ratio (PLR); neutrophil-tolymphocyte ratio (NLR); prognosis; meta-analysis

Submitted Feb 14, 2020. Accepted for publication Aug 05, 2020.

doi: $10.21037 /$ tcr-20-1037

View this article at: http://dx.doi.org/10.21037/tcr-20-1037

\section{Introduction}

Gastrointestinal stromal tumours (GISTs) are the most common mesenchymal tumours of the gastrointestinal tract and account for approximately $0.2 \%$ of all GI malignancies (1). They are deemed to arise from the interstitial cells of Cajal (ICC) and are composed of predominantly spindle or epithelioid cells $(2,3)$. GISTs are found primarily in the stomach $(60-70 \%)$ and the small intestine $(30 \%)$ but may also occur in the colon, rectum, 
and oesophagus (4). The tumorigenesis and progression of GISTs are partially attributed to gain-of-function mutations in the KIT proto-oncogene or platelet-derived growth factor receptor alpha (PDGFR $\alpha$ ) gene, which serve as therapeutic targets for targeted therapy (5). R0 resection is the first treatment option for localized GISTs (6-9). However, some patients still develop recurrent disease even after R0 resection (10). It is critical to identify factors that predict the treatment response and survival of GIST patients.

Presently, most of the well-established prognostic factors of GISTs rely heavily on the results of tissue biopsy, including the mitotic index, tumour size, location of the primary tumour and tumour rupture $(11,12)$. However, there is a gap between estimated recurrence rates by risk-stratification models and the exact recurrence rates (13). Therefore, it is critical to develop novel criteria or supplement the current stratification systems with new parameters.

Over the past decade, it has been elucidated that systemic inflammation could promote tumour metastasis by inducing angiogenesis or inhibiting apoptosis $(14,15)$. Inflammationbased biomarkers, such as the platelet-to-lymphocyte ratio (PLR) and neutrophil-to-lymphocyte ratio (NLR), have been demonstrated to be independent prognostic factors in several types of tumours (16-19). Recently, several studies have been performed to assess the prognostic value of PLR and NLR in GISTs. One meta-analysis suggested that NLR is an independent prognostic factor in patients with GISTs (20). Based on their results, the prognostic effects of PLR and NLR in GISTs remain inconsistent. Therefore, we performed this systematic analysis to assess the prognostic effects of PLR and NLR in GISTs. We present the following article in accordance with the PRISMA reporting checklist (available at http://dx.doi.org/10.21037/tcr-20-1037).

\section{Methods}

\section{Search strategy}

We performed a comprehensive search in MEDLINE, EMBASE and the Cochrane Library. The protocol for this systematic review was registered on PROSPERO (ID: CRD42020168505) and is available in full on the University of York website. The following terms were searched: "gastrointestinal stromal tumors" or "GISTs", "PLR" (or "platelet-lymphocyte ratio") or "NLR" (or "neutrophil-lymphocyte ratio"), "survival" or "prognostic" or "prognosis" or "recurrence" or "clinical outcome" (Figure 1).

\section{Study selection}

The inclusion criteria included the following: (I) all patients had been diagnosed with GIST by pathological examination; (II) the association between PLR and overall survival (OS) and/or disease-free survival (DFS)/recurrencefree survival (RFS) or between NLR and OS and/or DFS/RFS was evaluated; and (III) all blood samples were obtained before treatment. The following types of studies were excluded: (I) case reports, reviews, conference abstracts and letters; (II) studies with insufficient data to calculate an HR and $95 \%$ CI; and (III) overlapping or duplicate publications. If multiple studies were reported by the same team from the same institute or were performed at the same time, only the latest article was included.

\section{Data extraction}

Data were reviewed and extracted independently by two authors (ZW Wei and WB Huang). The following information was recorded from each study: first author, study design, year of publication, country, number of subjects, NIH risk categories, PLR and NLR cut-off values, time of follow-up, outcome measures (HRs for OS and DFS/RFS and their 95\% CIs), and clinicopathological characteristics. Hazard ratios were provided by the original studies or could be estimated from Kaplan-Meier survival curves (21).

\section{Quality assessment}

Study quality assessment was performed independently by two investigators according to the Newcastle-Ottawa Scale (NOS) (22). The range of total scores was from 0 to 9. High-quality trials scored more than 6.

\section{Statistical analysis}

The log HR and standard error (SE) were used to pool the survival results $(21,23)$. Cochran's $Q$ test and the $\mathrm{I}^{2}$ statistic were used to evaluate the heterogeneity of the pooled outcomes. A P value $<0.1$ for the Q-test or $\mathrm{I}^{2}>50 \%$ suggested significant heterogeneity among the included studies, and a random-effects model was used. A fixed-effects model was used to estimate the effect 


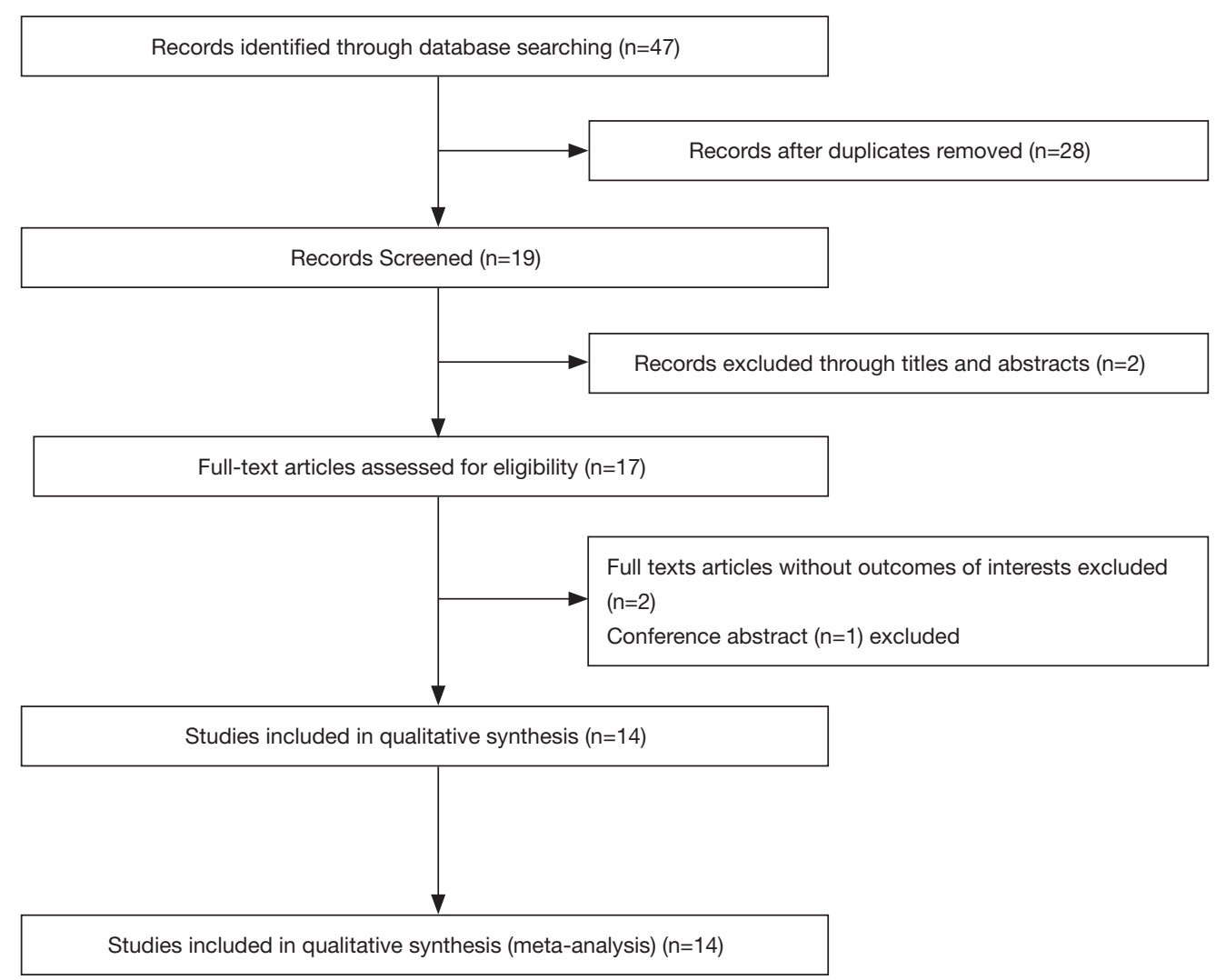

Figure 1 Systematic search and selection strategy.

magnitude when inter-study heterogeneity was absent. Subgroup analyses were performed according to the area of publication, sample size, treatment, analysis method, cut-off value of PLR and NOS score. Publication bias was analysed by assessing the symmetry of the funnel plot and performing Egger's test. The effect of potentially unpublished studies was investigated using the trim and fill procedure. Statistical analysis was performed with Review Manager Version 5.2 (Nordic Cochrane Centre, the Cochrane Collaboration), Stata version 14 and the $\mathrm{R}$ software environment.

\section{Results}

\section{Description of studies}

The initial search strategy identified 47 articles, and 28 articles were excluded after removing duplicate articles (Figure 1). Fourteen studies published between 2013 and 2019 including 3151 GIST patients were enrolled in this meta-analysis (Table 1) $(13,24-36)$. Eight studies assessed the prognostic impact of both PLR and NLR, and five of the enrolled studies investigated only NLR.

The number of subjects in each study varied from 67 to 510 . Seven studies were from China, 2 were from Poland, 1 was from Singapore, 1 was from Canada, 1 was from Turkey, 1 was from the USA and 1 was from Austria. Thirteen studies determined DFS, and only 2 studies reported OS. Nine of the studies had $\geq 200$ patients, and the other five had $<200$ patients (Table 1).

\section{Prognostic impact of PLR and NLR}

A total of 9 studies including 2,860 patients investigated the association between PLR and DFS. Significant heterogeneity was detected among the 9 studies $\left(\mathrm{I}^{2}=64 \%\right.$, $\mathrm{P}=0.002$ ) (Figure $S 1 A$ ). The heterogeneity seemed to be attributed to the study by Goh et al. After excluding this study, the heterogeneity decreased $\left(\mathrm{P}=0.38, \mathrm{I}^{2}=7 \%\right)$, and DFS was still significantly worse for the high PLR group than for the low PLR group, with a pooled HR of 1.29 (95\% CI: $1.10-1.52, \mathrm{P}=0.002$; Figure $2 A$ ).

Twelve studies reported the impact of NLR on DFS/RFS 


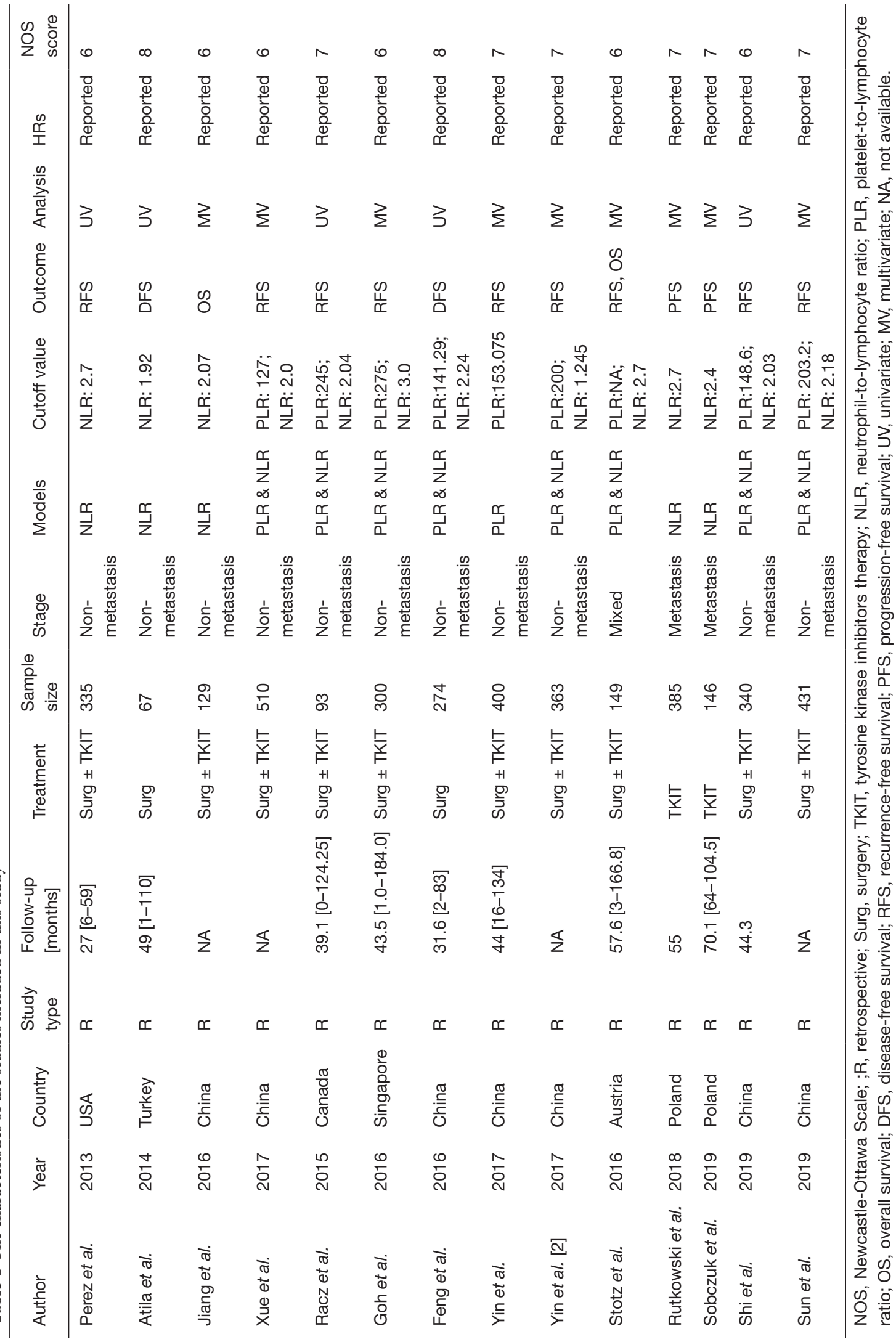




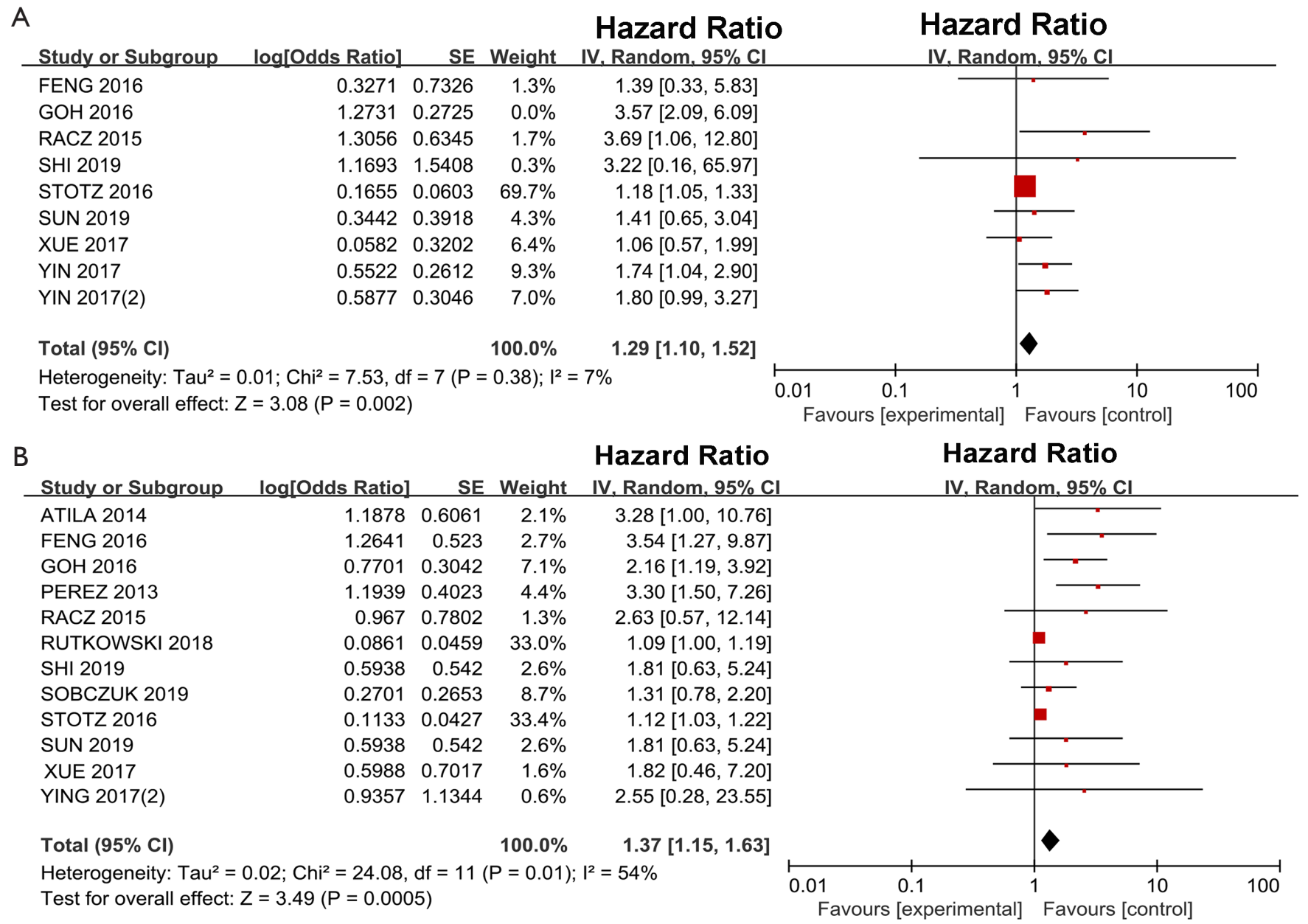

Figure 2 Forest plots of (A) platelet-to-lymphocyte ratio (PLR) and (B) neutrophil-to-lymphocyte ratio (NLR) in predicting disease-free survival.

in GISTs. As shown in Figure 2B, the pooled analysis of the 12 studies showed that the DFS of patients with a high NLR was significantly inferior to that of patients with a low NLR (HR $=1.37,95 \%$ CI: $1.15-1.63 ; \mathrm{P}=0.0005)$.

In addition, only two studies with 278 patients explored the prognostic effect of NLR on OS. The OS of the high NLR group seemed worse, but the difference was not significant (HR =1.74; $\mathrm{P}=0.29$ ) (Figure S1B). Only Stotz et al. (29) investigated the association between PLR and OS and revealed that high PLR is an unfavourable predictor of OS.

\section{Subgroup analysis of PLR for DFS}

In addition, we performed meta-regression and subgroup analyses by area, sample size, analysis method, PLR cutoff value and NOS score, as shown in Table 2. Elevated
PLR was correlated with poor DFS in studies performed in Eastern countries (HR =1.52, 95\% CI: 1.13-2.04; $\mathrm{P}=0.006$ ). In the subgroup analysis by sample size, a high PLR was associated with inferior DFS in studies with sample sizes $\geq 200(\mathrm{HR}=1.80,95 \% \mathrm{CI}: 1.27-2.57 ; \mathrm{P}=0.001)$. The prognostic impact of high PLR was significant regardless of the analysis method (univariate or multivariate). The cut-off values of PLR ranged from 127 to 275 . According to the cut-off value of PLR, studies were stratified into two subgroups: $<200$ and $\geq 200$. Stratification based on the cut-off value showed that a high PLR was associated with poor DFS in studies with cut-off values $\geq 200$ (HR $=2.08,95 \%$ CI: $1.04-4.15 ; \mathrm{P}=0.040)$. Moreover, subgroup analysis suggested that elevated PLR significantly predicted decreased DFS in studies with NOS scores $\geq 7$ (HR $=1.76$, 95\% CI: 1.27-2.44; $\mathrm{P}=0.0007$ ). 
Table 2 Pooled hazard ratios (HRs) for DFS/RFS according to subgroup analyses based on the platelet-to-lymphocyte ratio (PLR)

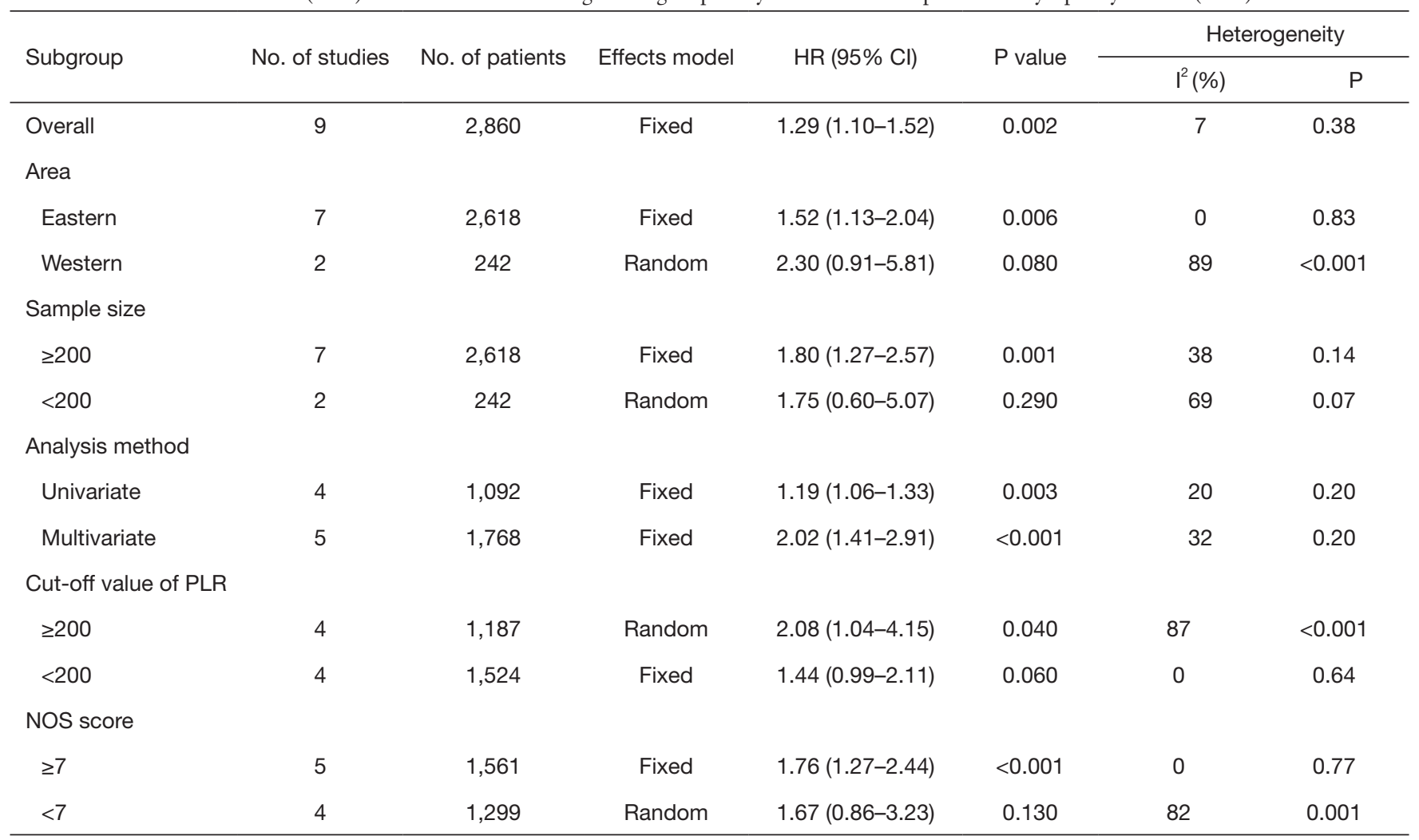

NOS, Newcastle-Ottawa Scale.

\section{Subgroup analysis of NLR for DFS}

Among all the studies on NLR, 5 studies were performed in Eastern countries, and 7 studies were conducted in Western countries (Table 3). Subgroup analysis suggested that the correlations between NLR and DFS were still significant in both the Eastern countries (HR $=2.22,95 \%$ CI: 1.30-3.81; $\mathrm{P}=0.004)$ and Western countries (HR $=1.28,95 \%$ CI: 1.07 1.53; $\mathrm{P}=0.006$ ) (Table 3). The results of the pooled analysis of 8 studies with sample sizes greater than 200 revealed that an elevated NLR was associated with poor survival (HR $=1.96,95 \%$ CI: $1.24-3.08 ; \mathrm{P}=0.004)$. Concerning the analysis method, the prognostic impact of NLR on DFS was still significant according to univariate and multivariate analyses. Subgroup analyses stratified by NOS score showed that high NLR was correlated with shorter DFS regardless of NOS score.

\section{Associations between PLR, NLR and clinicopathological features}

The present meta-analysis explored the effect of PLR on 7 clinical factors previously identified in GISTs. The pooled analysis demonstrated that elevated PLR was correlated with larger tumour size $(>5$ vs. $<5 \mathrm{~cm}$; OR $=2.46,95 \% \mathrm{CI}$ : 1.87-3.22; $\mathrm{P}<0.001)$, mitotic index $(>5 / 50 \mathrm{HPF} v s .<5 / 50$ HPF; OR $=2.09,95 \%$ CI: $1.61-2.71 ; \mathrm{P}<0.001)$, adjuvant tyrosine kinase inhibitor (TKI) therapy (yes vs. no; OR $=5.05,95 \%$ CI: $1.07-23.75 ; \mathrm{P}=0.04)$, and NIH risk category (high/intermediate $v s$. very low/low; OR $=2.72,95 \% \mathrm{CI}$ : 2.03-3.64; $\mathrm{P}<0.001)$. Meanwhile, no significant association was found for sex (male $v s$. female), primary tumour site (stomach $v s$. non-stomach) or cellular type (spindle $v s$. non-spindle). The correlations between PLR and the clinicopathological features of GISTs are shown in Table S1.

As shown in Table S2, the correlations between NLR and clinicopathological features were also investigated. Similarly, a high NLR can predict the prognosis of patients with GISTs larger than $5 \mathrm{~cm}(\mathrm{OR}=1.91,95 \%$ CI: $1.48-2.47$; $\mathrm{P}<0.001)$ and those in the high/intermediate risk groups (OR $=2.41,95 \%$ CI: $1.49-3.89 ; \mathrm{P}<0.001)$. Moreover, elevated NLR was correlated with male sex and mitotic index $(>5 / 50$ HPF $v s .<5 / 50$ HPF; OR $=1.80 ; \mathrm{P}=0.006$ ). 
Table 3 Pooled hazard ratios (HRs) for DFS/RFS according to subgroup analyse based on neutrophil-to-lymphocyte ratio (NLR)

\begin{tabular}{|c|c|c|c|c|c|c|c|}
\hline Subgroup & No. of studies & $\begin{array}{c}\text { No. of } \\
\text { patients }\end{array}$ & Effects model & $\mathrm{HR}(95 \% \mathrm{Cl})$ & $P$ value & \multicolumn{2}{|c|}{ Heterogeneity } \\
\hline Overall & 12 & 3,393 & Fixed & $1.37(1.15-1.63)$ & 0.0005 & 54 & 0.01 \\
\hline \multicolumn{8}{|l|}{ Area } \\
\hline Eastern & 5 & 1,918 & Fixed & $2.22(1.30-3.81)$ & 0.004 & 0 & 0.88 \\
\hline \multicolumn{8}{|l|}{ Sample size } \\
\hline$\geq 200$ & 8 & 2,938 & Random & $1.96(1.24-3.08)$ & 0.004 & 64 & 0.007 \\
\hline$<200$ & 4 & 455 & Fixed & $1.31(0.93-1.83)$ & 0.12 & 35 & 0.20 \\
\hline \multicolumn{8}{|c|}{ Analysis method } \\
\hline \multicolumn{8}{|l|}{ NOS score } \\
\hline$\geq 7$ & 7 & 1,759 & Fixed & $1.61(1.09-2.38)$ & 0.02 & 46 & 0.08 \\
\hline$<7$ & 5 & 1,634 & Random & $1.80(1.08-3.00)$ & 0.02 & 68 & 0.01 \\
\hline
\end{tabular}

\section{Comparison between PLR and NLR}

Presently, PLR and NLR are the two most widely available markers in GISTs (25). Seven and four studies reported the HR for PLR and NLR in univariate and multivariate analyses, respectively. The pooled HR for PLR was not significantly different from that for NLR (Table S3).

\section{Publication bias}

Funnel plots of PLR and NLR were used to evaluate publication bias, as shown in Figure 3. Then, the asymmetry of the funnel plots was further tested by Egger's test. No evidence of obvious publication bias was detected by Egger's test $(\mathrm{P}=0.072)$ for PLR. For NLR, significant publication bias was found by Egger's test $(\mathrm{P}<0.001)$. Then, a trim and fill analysis was performed to investigate the potential effect of publication bias. After incorporating 7 hypothetical studies, the funnel plots were shown to be symmetrical (Figure 4). The adjusted pooled analysis with the 7 additional studies showed that the DFS of patients with a high NLR was still worse than that of patients with a low NLR (HR $=1.19,95 \%$ CI: $1.01-1.42$ ).

\section{Discussion}

Emerging evidence has demonstrated that inflammation- based factors, including PLR and NLR, which are representative of the inflammatory response, are associated with worse outcomes in several malignant tumours (37). Several studies demonstrated that PLR and NLR are independent prognostic factors in GISTs, while some other studies did not find similar results. A meta-analysis performed by Luo et al. suggested that an elevated preoperative NLR is associated with unfavourable outcomes in patients with GISTs (20). However, the prognostic role of PLR and NLR in GISTs remains controversial. We enrolled 14 studies involving 3,151 patients that investigated the prognostic roles of PLR and NLR in patients with GISTs. This meta-analysis aimed to identify the effects of elevated PLR and NLR on prognosis in patients with GISTs.

In the present meta-analysis, we demonstrated that elevated PLR and NLR are associated with worse DFS in GISTs. Although the specific mechanism remains unclear, our results are consistent with other studies that demonstrated that PLR or NLR was predictive of poorer prognosis in multiple types of malignancies, including colorectal cancer, hepatocellular carcinoma, and oesophageal tumours (38-42). PLR and NLR indicate the levels of systematic inflammation, which play vital roles in tumour progression $(43,44)$. Platelets can release a variety of cytokines and chemokines to promote tumour metastasis (45). Labelle et al. reported that platelets recruit 

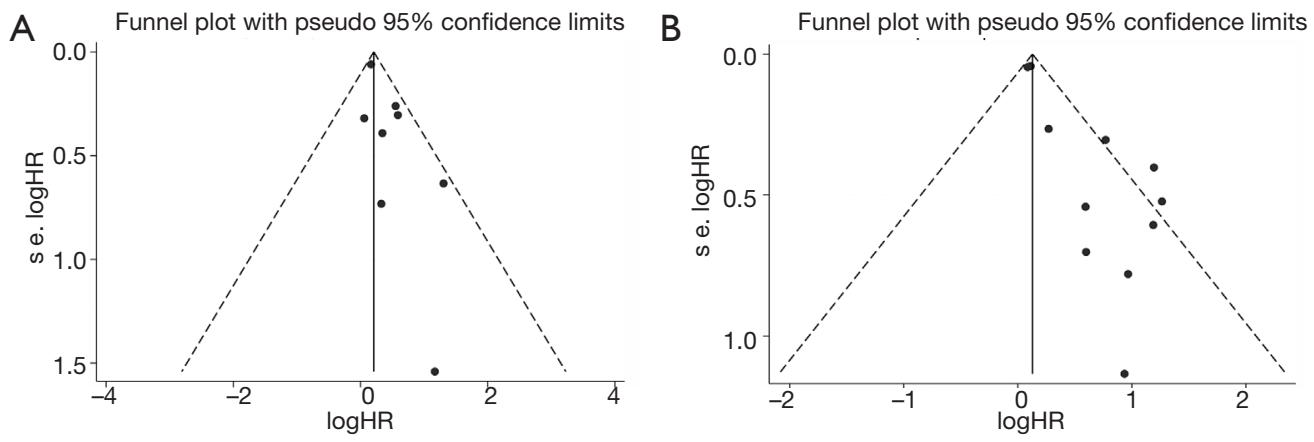

Figure 3 Funnel plots of DFS for (A) platelet-to-lymphocyte ratio (PLR) and (B) neutrophil-to-lymphocyte ratio (NLR).

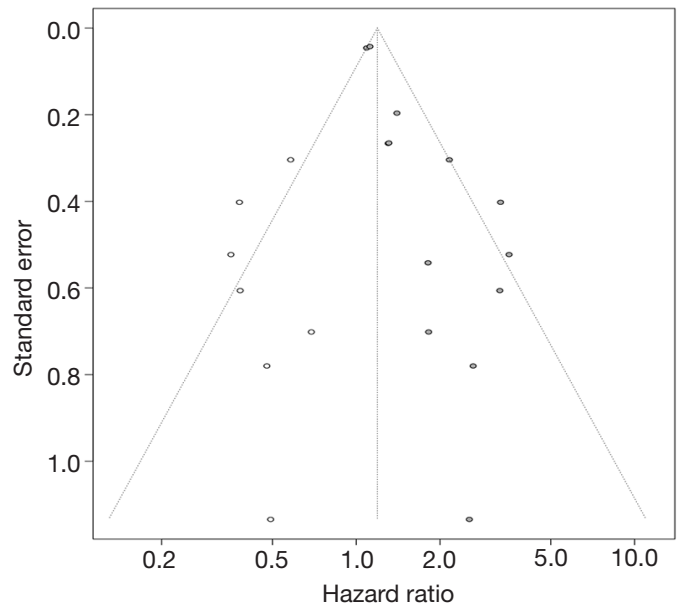

Figure 4 Funnel plots with trim-and-fill analysis for neutrophilto-lymphocyte ratio (NLR).

granulocytes and facilitate the formation of premetastatic niches, which are critical for metastasis (46). Moreover, there is increasing evidence suggesting crosstalk between platelets and tumour cells that contributes to tumour growth and progression (47). Lymphocytopenia and the suppression of lymphocyte activity induced by the systemic inflammatory response may result in the impairment of innate cellular immunity, leading to inferior survival (48). Given their important roles, elevated PLR combined with the effects of thrombocytosis and lymphocytopenia may be associated with the prognosis of GISTs. Moreover, it is well established that neutrophils act as key regulators of tumour-associated inflammation and the immune response (49). Neutrophils, especially tumourassociated neutrophils, are recruited into the tumour microenvironment by cytokines and chemokines to regulate inflammation, immunosuppression and tumour growth (50). Moreover, neutrophils counteract the function of immune cells to facilitate tumorigenesis via reactive oxygen species (ROS) and arginase-I (ARG) (51). Thus, NLR, as a marker of neutrophilia and lymphocytopenia, is correlated with poor prognosis in GISTs.

Subgroup analysis revealed that elevated PLR and NLR remain significant prognostic factors of DFS/RFS for GISTs in studies with sample sizes $\geq 200$. The nonsignificant associations between PLR, NLR and DFS may be due to the limited number of studies with fewer than 200 subjects. Given that the cut-off values of PLR varied among the included studies, we performed subgroup analysis to assess the effects of different cut-off values on the prognostic impact of PLR and demonstrated that patients with elevated PLR suffer worse prognosis than those with low PLR in studies with cut-off values $\geq 200$. Subgroup analysis of PLR for DFS revealed that no differences could distinctly be observed in Western countries. However, the limited sample size, with only 242 subjects from Western countries, might be responsible for this result. For NLR, significant differences were detected in both Eastern and Western countries. Moreover, elevated PLR and NLR predicted worse DFS/RFS in patients with GISTs, regardless of the analysis method.

PLR and NLR reflect the systemic inflammatory status, which is a hallmark of tumours. Yang et al. reported that PLR is elevated with TNM stage in colon cancers (52). Our data suggested that patients with high PLR tend to fall into the NIH high- and intermediate-risk categories. Liu et al. reported that elevated NLR is associated with increased tumour size in thyroid cancer (53). Consistent with the studies mentioned above, the present meta-analysis demonstrated that GISTs larger than $5 \mathrm{~cm}$ seem to correlate 
with high PLR and NLR. The innate immune system in early-stage tumours can recognize and eliminate tumour cells, and afterwards, with the progression of tumours, tumour cells can evade immune surveillance, leading to more advanced tumours (54). Hence, high PLR and NLR, indicators of systemic inflammation, correlate with advanced tumour stages in GISTs.

For GISTs, PLR and NLR are currently the two most widely available inflammatory markers. Racz et al. demonstrated that only PLR but not NLR is associated with prognosis in GISTs by univariate analysis (24). Goh et al. concluded that both PLR and NLR are independent prognostic factors in GISTs (25). Herein, we attempted to compare the prognostic influence of PLR and NLR, and no significant difference was observed.

Nevertheless, several limitations need to be addressed in the current meta-analysis. First, significant heterogeneity existed among the studies. The difference in cutoff values may be the major reason for the heterogeneity. Second, all the included studies were retrospective, and there were no randomized controlled trials (RCTs). Given the retrospective nature of the enrolled studies, the magnitude of any confounding factors would have been further amplified, and original articles supplied only summarized but not original data, which may have increased the heterogeneity. Third, HRs and their 95\% CIs were extracted from univariable analysis in five studies. Consequently, the prognostic effect of PLR and NLR might be overestimated.

\section{Conclusions}

The current meta-analysis demonstrated that elevated preoperative PLR and NLR play significant roles in the prognosis of patients with GISTs. Additionally, PLR and NLR are cost-effective markers that may serve as potential prognostic biomarkers for GISTs in clinical practice. More well-designed and high-quality multicentre clinical trials are warranted to validate the impact of PLR and NLR in risk stratifications.

\section{Acknowledgments}

Funding: This study was supported by the National Natural Science Foundation of China (81702325) and natural Science Foundation of Guangdong Province (2017A030310565).

\section{Footnote}

Reporting Checklist: The authors have completed the PRISMA reporting checklist. Available at http://dx.doi. org/10.21037/tcr-20-1037

Conflicts of Interest: All authors have completed the ICMJE uniform disclosure form (available at http://dx.doi. org/10.21037/tcr-20-1037). The authors have no conflicts of interest to declare.

Ethical Statement: The authors are accountable for all aspects of the work in ensuring that questions related to the accuracy or integrity of any part of the work are appropriately investigated and resolved.

Open Access Statement: This is an Open Access article distributed in accordance with the Creative Commons Attribution-NonCommercial-NoDerivs 4.0 International License (CC BY-NC-ND 4.0), which permits the noncommercial replication and distribution of the article with the strict proviso that no changes or edits are made and the original work is properly cited (including links to both the formal publication through the relevant DOI and the license). See: https://creativecommons.org/licenses/by-nc-nd/4.0/.

\section{References}

1. Schrage Y, Hartgrink H, Smith M, et al. Surgical management of metastatic gastrointestinal stromal tumour. Eur J Surg Oncol 2018;44:1295-300.

2. Miettinen M, Lasota J. Gastrointestinal stromal tumors. Gastroenterol Clin North Am 2013;42:399-415.

3. Mei L, Smith SC, Faber AC, et al. Gastrointestinal Stromal Tumors: The GIST of Precision Medicine. Trends Cancer 2018;4:74-91.

4. De Vogelaere K, Van Loo I, Peters O, et al. Laparoscopic resection of gastric gastrointestinal stromal tumors (GIST) is safe and effective, irrespective of tumor size. Surg Endosc 2012;26:2339-45.

5. Mei L, Du W, Idowu M, et al. Advances and Challenges on Management of Gastrointestinal Stromal Tumors. Front Oncol 2018;8:135.

6. Koh YX, Chok AY, Zheng HL, et al. A systematic review and meta-analysis comparing laparoscopic versus open gastric resections for gastrointestinal stromal tumors of the stomach. Ann Surg Oncol 2013;20:3549-60.

7. Goh BK, Chow PK, Kesavan SM, et al. Outcome 
after curative resection of large $(>\mathrm{OR}=10 \mathrm{~cm})$ gastric gastrointestinal stromal tumors: how frequent is adjacent organ involvement and is concomitant distal pancreatectomy necessary? J Gastrointest Surg 2010;14:607-13.

8. Chok AY, Koh YX, Ow MY, et al. A systematic review and meta-analysis comparing pancreaticoduodenectomy versus limited resection for duodenal gastrointestinal stromal tumors. Ann Surg Oncol 2014;21:3429-38.

9. Nishida T, Blay JY, Hirota S, et al. The standard diagnosis, treatment, and follow-up of gastrointestinal stromal tumors based on guidelines. Gastric Cancer 2016;19:3-14.

10. Hølmebakk T, Hompland I, Bjerkehagen B, et al. Recurrence-Free Survival After Resection of Gastric Gastrointestinal Stromal Tumors Classified According to a Strict Definition of Tumor Rupture: A Population-Based Study. Ann Surg Oncol 2018;25:1133-9.

11. Yanagimoto Y, Takahashi T, Muguruma K, et al. Reappraisal of risk classifications for primary gastrointestinal stromal tumors (GISTs) after complete resection: indications for adjuvant therapy. Gastric Cancer 2015;18:426-33.

12. Joensuu H, Vehtari A, Riihimaki J, et al. Risk of recurrence of gastrointestinal stromal tumour after surgery: an analysis of pooled population-based cohorts. Lancet Oncol 2012;13:265-74.

13. Xue A, Gao X, Fang Y, et al. Incorporation of NLR into NIH stratification system increases predictive accuracy for surgically resected gastrointestinal stromal tumors. Acta Biochim Biophys Sin (Shanghai) 2017;49:179-85.

14. Shalapour S, Karin M. Immunity, inflammation, and cancer: an eternal fight between good and evil. J Clin Invest 2015;125:3347-55.

15. Diakos CI, Charles KA, McMillan DC, et al. Cancerrelated inflammation and treatment effectiveness. Lancet Oncol 2014;15:e493-503.

16. Paramanathan A, Saxena A, Morris DL. A systematic review and meta-analysis on the impact of pre-operative neutrophil lymphocyte ratio on long term outcomes after curative intent resection of solid tumours. Surg Oncol 2014;23:31-9.

17. Pierce BL, Ballard-Barbash R, Bernstein L, et al. Elevated biomarkers of inflammation are associated with reduced survival among breast cancer patients. J Clin Oncol 2009;27:3437-44.

18. Zhou X, Du Y, Huang Z, et al. Prognostic value of PLR in various cancers: a meta-analysis. PLoS One 2014;9:e101119.
19. Templeton AJ, McNamara MG, Seruga B, et al. Prognostic role of neutrophil-to-lymphocyte ratio in solid tumors: a systematic review and meta-analysis. J Natl Cancer Inst 2014;106:dju124.

20. Luo XF, Zhou LH. Prognostic significance of neutrophil to lymphocyte ratio in patients with gastrointestinal stromal tumors: A meta-analysis. Clin Chim Acta 2018;477:7-12.

21. Parmar MK, Torri V, Stewart L. Extracting summary statistics to perform meta-analyses of the published literature for survival endpoints. Stat Med 1998;17:2815-34.

22. Stang A. Critical evaluation of the Newcastle-Ottawa scale for the assessment of the quality of nonrandomized studies in meta-analyses. Eur J Epidemiol 2010;25:603-5.

23. Tierney JF, Stewart LA, Ghersi D, et al. Practical methods for incorporating summary time-to-event data into metaanalysis. Trials 2007;8:16.

24. Racz JM, Cleghorn MC, Jimenez MC, et al. Predictive Ability of Blood Neutrophil-to-Lymphocyte and Plateletto-Lymphocyte Ratios in Gastrointestinal Stromal Tumors. Ann Surg Oncol 2015;22:2343-50.

25. Goh BK, Chok AY, Allen JC Jr, et al. Blood neutrophilto-lymphocyte and platelet-to-lymphocyte ratios are independent prognostic factors for surgically resected gastrointestinal stromal tumors. Surgery 2016;159:1146-56.

26. Feng F, Tian Y, Liu S, et al. Combination of PLR, MLR, MWR, and Tumor Size Could Significantly Increase the Prognostic Value for Gastrointestinal Stromal Tumors. Medicine (Baltimore) 2016;95:e3248.

27. Yin XN, Tang SM, Yin Y, et al. [Associations of Preoperative Platelet-to-lymphocyte Ratio and Derived Neutrophil-to-lymphocyte Ratio with thePrognosis of Gastrointestinal Stromal Tumor]. Sichuan Da Xue Xue Bao Yi Xue Ban 2017;48:239-43.

28. Yin Z, Gao J, Liu W, et al. Clinicopathological and Prognostic Analysis of Primary Gastrointestinal Stromal Tumor Presenting with Gastrointestinal Bleeding: a 10-Year Retrospective Study. J Gastrointest Surg 2017;21:792-800.

29. Stotz M, Liegl-Atzwanger B, Posch F, et al. Blood-Based Biomarkers Are Associated with Disease Recurrence and Survival in Gastrointestinal Stroma Tumor Patients after Surgical Resection. PLoS One 2016;11:e0159448.

30. Shi WK, Zhang XH, Zhang J, et al. Predictive ability of prognostic nutritional index in surgically resected gastrointestinal stromal tumors: a propensity score matching analysis. Jpn J Clin Oncol 2019;49:823-31.

31. Sun J, Mei Y, Zhu Q, et al. Relationship of prognostic nutritional index with prognosis of gastrointestinal stromal 
tumors. J Cancer 2019;10:2679-86.

32. Perez DR, Baser RE, Cavnar MJ, et al. Blood neutrophilto-lymphocyte ratio is prognostic in gastrointestinal stromal tumor. Ann Surg Oncol 2013;20:593-9.

33. Atila K, Arslan NC, Derici S, et al. Neutrophil-tolymphocyte ratio: could it be used in the clinic as prognostic marker for gastrointestinal stromal tumor? Hepatogastroenterology 2014;61:1649-53.

34. Jiang C, Hu WM, Liao FX, et al. Elevated preoperative neutrophil-to-lymphocyte ratio is associated with poor prognosis in gastrointestinal stromal tumor patients. Onco Targets Ther 2016;9:877-83.

35. Sobczuk P, Teterycz P, Lugowska I, et al. Prognostic value of the pretreatment neutrophil-to-lymphocyte ratio in patients with advanced gastrointestinal stromal tumors treated with sunitinib after imatinib failure. Oncol Lett 2019;18:3373-80.

36. Rutkowski P, Teterycz P, Klimczak A, et al. Blood neutrophil-to-lymphocyte ratio is associated with prognosis in advanced gastrointestinal stromal tumors treated with imatinib. Tumori 2018;104:415-22.

37. Templeton AJ, Ace O, McNamara MG, et al. Prognostic role of platelet to lymphocyte ratio in solid tumors: a systematic review and meta-analysis. Cancer Epidemiol Biomarkers Prev 2014;23:1204-12.

38. Yodying H, Matsuda A, Miyashita M, et al. Prognostic Significance of Neutrophil-to-Lymphocyte Ratio and Platelet-to-Lymphocyte Ratio in Oncologic Outcomes of Esophageal Cancer: A Systematic Review and Metaanalysis. Ann Surg Oncol 2016;23:646-54.

39. Szkandera J, Pichler M, Absenger G, et al. The elevated preoperative platelet to lymphocyte ratio predicts decreased time to recurrence in colon cancer patients. Am J Surg 2014;208:210-4.

40. Dell'Aquila E, Cremolini C, Zeppola T, et al. Prognostic and predictive role of neutrophil/lymphocytes ratio in metastatic colorectal cancer: a retrospective analysis of the TRIBE study by GONO. Ann Oncol 2018;29:924-30.

41. Zhang Y, Brodin NP, Ohri N, et al. Association between neutrophil-lymphocyte ratio, socioeconomic status, and ethnic minority with treatment outcome in hepatocellular carcinoma. Hepatol Int 2019;13:609-17.

42. Ishibashi Y, Tsujimoto H, Hiraki S, et al. Prognostic Value of Preoperative Systemic Immunoinflammatory Measures in Patients with Esophageal Cancer. Ann Surg Oncol 2018;25:3288-99.

43. Grinberg-Bleyer Y, Ghosh S. A Novel Link between
Inflammation and Cancer. Cancer Cell 2016;30:829-30.

44. Dong CH, Wang ZM, Chen SY. Neutrophil to lymphocyte ratio predict mortality and major adverse cardiac events in acute coronary syndrome: A systematic review and meta-analysis. Clin Biochem 2018;52:131-6.

45. Tesfamariam B. Involvement of platelets in tumor cell metastasis. Pharmacol Ther 2016;157:112-9.

46. Labelle M, Begum S, Hynes RO. Platelets guide the formation of early metastatic niches. Proc Natl Acad Sci U S A 2014;111:E3053-61.

47. Lonsdorf AS, Kramer BF, Fahrleitner M, et al. Engagement of alphaIIbbeta3 (GPIIb/IIIa) with alphanubeta3 integrin mediates interaction of melanoma cells with platelets: a connection to hematogenous metastasis. J Biol Chem 2012;287:2168-78.

48. Schalk E, Zeremski V, Fischer T. Impact of lymphopenia on prognosis of patients with primary central nervous system lymphoma. Eur J Cancer 2017;75:280-3.

49. Masucci MT, Minopoli M, Carriero MV. Tumor Associated Neutrophils. Their Role in Tumorigenesis, Metastasis, Prognosis and Therapy. Front Oncol 2019;9:1146.

50. Shaul ME, Fridlender ZG. Cancer-related circulating and tumor-associated neutrophils - subtypes, sources and function. FEBS J 2018;285:4316-42.

51. Zhang $\mathrm{X}, \mathrm{Xu}$ W. Neutrophils diminish T-cell immunity to foster gastric cancer progression: the role of GM-CSF/ PD-L1/PD-1 signalling pathway. Gut 2017;66:1878-80.

52. Yang L, He W, Kong $\mathrm{P}$, et al. Clinical baseline and prognostic difference of platelet lymphocyte ratio (PLR) in right-sided and let-sided colon cancers. BMC Cancer 2017;17:873.

53. Liu CL, Lee JJ, Liu TP, et al. Blood neutrophil-tolymphocyte ratio correlates with tumor size in patients with differentiated thyroid cancer. J Surg Oncol 2013;107:493-7.

54. Milette S, Fiset PO, Walsh LA, et al. The innate immune architecture of lung tumors and its implication in disease progression. J Pathol 2019;247:589-605.

Cite this article as: Wei ZW, Huang WB, Yang DJ, Yuan YJ, He YL, Zhang CH. The prognostic roles of plateletto-lymphocyte ratio and neutrophil-to-lymphocyte ratio in gastrointestinal stromal tumours: a meta-analysis. Transl Cancer Res 2020;9(9):5128-5138. doi: 10.21037/tcr-20-1037 
Supplementary

A

Hazard Ratio

Hazard Ratio

Study or Subgroup

log[Odds Ratio]

SE Weight

IV. Random, $95 \% \mathrm{Cl}$

IV. Random. $95 \% \mathrm{Cl}$

FENG 2016

GOH 2016

$\begin{array}{lll}0.3271 & 0.7326 \quad 4.5 \%\end{array}$

$\begin{array}{ll}1.2731 & 0.2725\end{array}$

RACZ 2015

$1.3056 \quad 0.6345$

$14.6 \%$

SHI 2019

STOTZ 2016

1.16931 .5408

$5.6 \%$

$1.39[0.33,5.83]$

$3.57[2.09,6.09]$

$3.69[1.06,12.80]$

$3.22[0.16,65.97]$

$1.18[1.05,1.33]$

$0.1655 \quad 0.0603 \quad 22.2 \%$

SUN 2019

$\begin{array}{lll}0.3442 & 0.3918 \quad 10.6 \%\end{array}$

XUE 2017

YIN 2017

YIN 2017(2)

$0.0582 \quad 0.3202 \quad 12.8 \%$

$\begin{array}{lll}0.5522 & 0.2612 \quad 15.0 \%\end{array}$

$1.41[0.65,3.04]$

$1.06[0.57,1.99]$

$1.74[1.04,2.90]$

$0.5877 \quad 0.3046 \quad 13.4 \%$

$1.80[0.99,3.27]$

Total $(95 \% \mathrm{Cl})$

$100.0 \% \quad 1.70[1.21,2.39]$

Heterogeneity: $\mathrm{Tau}^{2}=0.13 ; \mathrm{Chi}^{2}=22.18, \mathrm{df}=8(P=0.005) ; \mathrm{I}^{2}=64 \%$

Test for overall effect: $Z=3.06(P=0.002)$

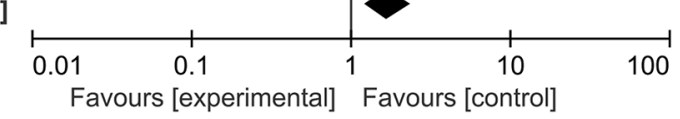

B

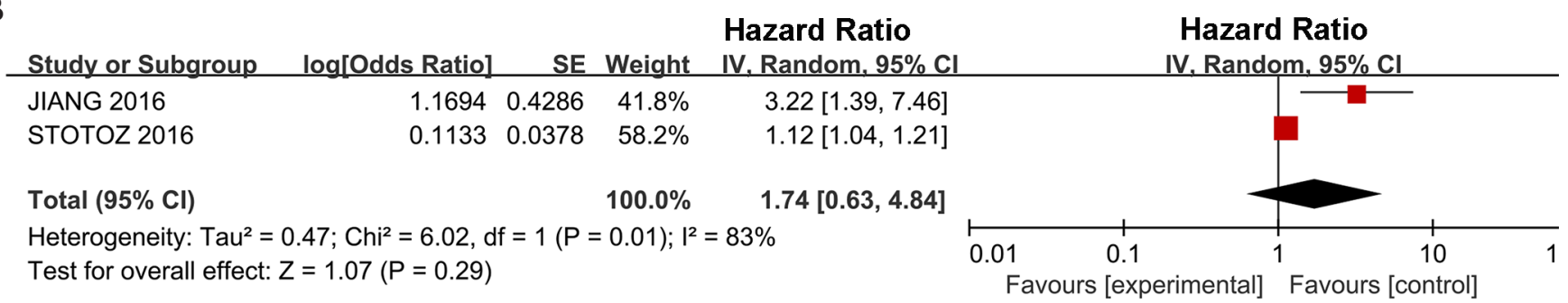

Figure S1 Forest plots of (A) platelet-to-lymphocyte ratio (PLR) in predicting DFS including the study of Goh et al. and (B) neutrophil-tolymphocyte ratio (NLR) in predicting OS.

Table S1 Meta-analysis of the association between platelet-to-lymphocyte ratio (PLR) and clinicopathological features of gastrointestinal stromal tumours (GISTs)

\begin{tabular}{|c|c|c|c|c|c|c|}
\hline Characteristics & No. of studies & $\begin{array}{l}\text { No. of pa- } \\
\text { tients }\end{array}$ & OR $(95 \% \mathrm{Cl})$ & $\mathrm{P}$ & \multicolumn{2}{|c|}{ Heterogeneity } \\
\hline Gender (male vs. female) & 5 & 1,407 & $1.14(0.76-1.71)$ & 0.52 & 54 & 0.09 \\
\hline Tumor size (>5 vs. $<5 \mathrm{~cm}$ ) & 5 & 1,406 & $2.46(1.87-3.22)$ & $<0.001$ & 0 & 0.65 \\
\hline $\begin{array}{l}\text { Primary tumor site (stomach vs. } \\
\text { non-stomach) }\end{array}$ & 5 & 1,407 & $0.85(0.27-2.66)$ & 0.78 & 93 & $<0.001$ \\
\hline Cellular type (spindle vs. non-spindle) & 1 & 274 & $0.68(0.25-1.85)$ & 0.45 & - & - \\
\hline Adjuvant TKI therapy (yes vs. no) & 4 & 1,133 & $5.05(1.07-23.75)$ & 0.04 & 91 & $<0.001$ \\
\hline $\begin{array}{l}\text { NIH risk category (high/intermediate } \\
\text { vs. very low/low) }\end{array}$ & 4 & 1,314 & $2.72(2.03-3.64)$ & $<0.001$ & 0 & 0.91 \\
\hline
\end{tabular}

Mitotic index, per 50 high-power field (HPF). OR, odds ratio; TKI, tyrosine kinase inhibitors. 
Table S2 Meta-analysis of the association between neutrophil-to-lymphocyte ratio (NLR) and clinicopathological features of gastrointestinal stromal tumours (GISTs)

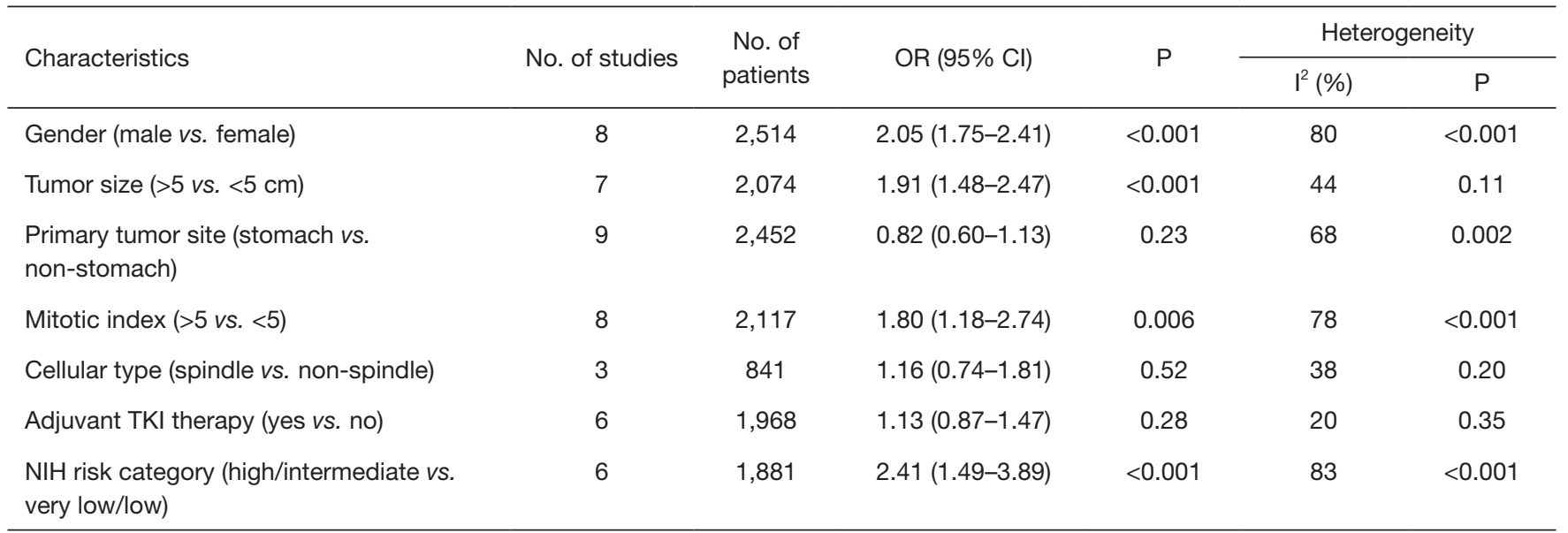

Mitotic index, per 50 high-power field (HPF). OR, odds ratio; TKI, tyrosine kinase inhibitors.

Table S3 Comparison of relative risk of HR between platelet-to-lymphocyte ratio (PLR) and neutrophil-to-lymphocyte ratio (NLR)

\begin{tabular}{lcccc}
\hline & Studies & Pooled HR for PLR (95\% Cl) & Pooled HR for NLR (95\% Cl) & Subgroup difference P \\
\hline PLR vs. NLR (univariate) & 7 & $2.568(1.385-4.760)$ & $2.409(1.725-3.365)$ & 0.858 \\
PLR vs. NLR (multivariate) & 4 & $1.892(1.282-2.792)$ & $2.307(1.432-3.716)$ & 0.528 \\
\hline
\end{tabular}

